\title{
ORGANIZING AND MANAGING VIRTUAL ENTERPRISES: THE ECB FRAMEWORK
}

\author{
Alessandro D'Atri \\ CeRSI - Luiss Guido Carli \\ datri@luiss.it \\ ITALY
}

This paper introduces a discourse about the organizational requirements of virtual enterprises (VEs). The growing economic globalization is threatening the competitiveness of traditional industrial districts that represent one of the building blocks of modern economy. VEs are efficient solutions to deal with uncertainty and complexity of the present competitive environment; in addition, they are suitable for reinforcing the global competitiveness of small and medium enterprise. To enable the incubation and creation of temporary networks of enterprises, a VE organizational framework is introduced. This framework is based on the role of three entities: Enabler, Catalyst and Broker (ECB). Main perceived benefits of the $E C B$ approach are: effective management of horizontal and vertical inter-relationships, efficient set-up of the VE, effective management of its operational phase.

\section{INTRODUCTION}

This paper introduces the main results of a research project aimed at identifying and evaluating organizational and technological solutions for innovative kinds of enterprises, in particular for virtual enterprises (a set of autonomous firms organically acting in a network system configured to exploit a specific business opportunity arising in the market).

The research activities focused on the identification of major classes of problems inhibiting the actual development of virtual enterprises. Three classes of inhibitors were recognized:

- SMEs' inertia in creatively applying the most advanced information and communication technologies: enterprises are used to deploy ICT to emphasize effectiveness and efficiency within single activities of their value chain. Hence, they usually fail to take advantage of the most revolutionary impact of ICT on organization: the integrated management of intensive interdependencies existing inside and outside the enterprise;

- Inefficient on-line environment for supporting the cooperation: SMEs willing to start a new virtual enterprise need on-line aggregation points where to meet each other, interact, find partners, and set up the venture. The lack of reliable 
and trustworthy on-line environments supplying added value services is hindering the development of virtual enterprises;

- Lack of predefined, efficient organizational configuration. Authors agree on the fact that there is no one best way for configuring a virtual enterprise (since it is shaped time-to-time in order to exploit the specific business opportunity as efficiently as possible). However, a general organizational framework would make the design and start-up phase of the cooperation much easier; in addition, it should allow an efficient management of the venture. In addition, interorganizational cooperation has to deal with the long standing problem of integration autonomous and distributed business processes (Venkatraman 1994). Many approaches to this integration have been proposed, but not one has imposed itself as generally effective (Davenport 1993) (Malone et al. 1999).

To match such inhibitors, a roadmap was developed. It is based on three classes of solutions:

- Technological solutions: the adoption of XML as a standard language for integrating distributed databases allows enterprise to consistently share data (first level of cooperation). The wide use of Internet technologies make easier to perform on-line transaction (e-commerce) with other enterprises (second level of cooperation); such technologies are also enabling the development of on-line relationships (e-business) among firm (third level of cooperation). In a foreseeable future, workflow management systems (WfMS) would make the integration of autonomous business processes possible. This latter form of cooperation would represent a major breakthrough in enabling virtual enterprises.

- Environmental solutions: an on-line environment, connected with an organizational structure has been developed. Such environment is based on the key role of a stable entity, the enabler.

- Organizational solutions: an organizational configuration founded on the complementary role of three subjects (enabler, catalyst and broker) has been introduced. Dynamic allocation of roles and high specialization are the keywords for describing this configuration.

The following sections, after briefly introducing the virtual enterprise paradigm, present a framework supporting the set-up, configuration and enactment of interorganizational business processes in the virtual enterprise. The framework proposes an organizational architecture potentially suitable to enable the development and management of virtual enterprises within a competitive sub-environment appropriately organized. A discussion and an outline of future research conclude the paper.

\section{VIRTUAL ENTERPRISES}

A virtual enterprise is a temporary network of autonomous enterprises based on the "plug and run" rule: single enterprises connect themselves stimulated and driven by the market - the network is configured as an efficient medium to exploit a specific business opportunity arising on the market. Every member makes available some proprietary sub-processes and part of its own knowledge. When the business opportunity is over, members disconnect and look for new businesses (Mowshowitz 
1997). Consisting in a set of SMEs, the virtual enterprise is usually informally organized and adopts non-hierarchical, lean and modular configurations. This kind of organizational architecture reflects the need to efficiently cooperate avoiding new bureaucracies and bottlenecks: the virtual enterprise is an adhocracy among organizations (Franke 2001).

The "planning" and "set up" phases of the virtual enterprise are very de-structured tasks, so it is quite difficult to formulate general rules and indicate a development path. Nevertheless, it is possible to identify a framework in which some entities perform a suitable set of activities. Main operative problems are related to the need for coordination among both members and processes (Malone and Crowston 1994) and can be summarized in terms of:

- Definition of the person/entity that envisions and plans the virtual enterprise: is it the firm identifying the market that selects the partners and integrates them? Is it realistic to believe that the enterprise that foresees the opportunity is naturally able to set-up and manage inter-organizational processes?

- Integration methods: does an "integrator entity" exist or is the virtual enterprise a completely bottom-up form of cooperation? Is it possible to perform an initial, normative phase in which the "rules" governing the virtual enterprise are established?

- The virtual business process: is it sufficient to collect the sub-processes supplied by members to obtain a common business process? Who is going to control and synchronize the virtual process? Is the virtual process just a trivial sequence of private sub-process or does it have a capability to be developed into more complex and inter-chained configurations?

\subsection{Typical Organizational Framework of Virtual Enterprises}

After the first wave of research and (mainly prototypical) deployment of virtual enterprises, it is now possible to analyze the typical organizational configuration applied.

In general, a central role in the management of a virtual enterprise has been assigned to a broker. The broker is viewed as necessary mainly because of the autonomy and the legal and economic independence characterizing the virtual cooperation: in a loosely coupled network, the presence of an integrator, i.e. of a subject able to coordinate and control it, becomes indispensable to catalyze single efforts towards the general goal. In addition, the broker has the awareness of the whole process of the virtual organization and it is capable, by applying its management capabilities together with its specialized knowledge, of allocating resources among the members and of managing the inter-organizational flows of information and knowledge.

Analyzing existing literature (Bremer1999) (Camarinha-Matos 2000), along with pilot applications (e.g. Projects Bidsaver, Cosme, Prodnet, Vive), it is evident that the configuration of the virtual enterprise based on an integrator (broker) is largely predominant.

Hence, according to the literature, the Virtual Enterprise broker (VEB) would act as:

- Initiator (it looks for the business opportunity and shapes the network);

- Coordinator (it manages and coordinate the virtual business process);

- Moderator (it is "a super partes" entity regulating the cooperation);

- Member of the VE (it is always a member of the network); 
- Information Broker (it integrates the members' autonomous information systems);

- Primary Point of Contact for Customers (it exploits the business opportunity and has the responsibility of customer management and marketing).

The VEB is responsible for the entire management and coordination of the virtual enterprise (from the business opportunity seeking phase to the dissolution of the network). Virtual Enterprises organized in accordance with such approach may be defined as "broker centered". Broker-centered Virtual Enterprises, while extremely common, cannot be universally considered as efficient solution. In the following, we are evidencing some major limitation of this configuration:

- Market exploitation inefficiency: the broker being the only entity in charge of scanning the market for arising opportunities, the whole network will have just one "eye" on the market. Many opportunities may be missing due to shortsighting of the broker. If, on the other side, member SMEs are entitled to look for opportunities and then have to pass the business to the broker for the management phase, a conflict of interests may arise: it is high probable that members will be not willing to lose their own customers in favor of the broker.

- Centralization: the broker becomes an essential player. If it is ineffective or it leaves the virtual enterprise, the network expires. In addition, the broker, as main general coordinator, can easily cause bottlenecks.

- High complexity: the activities related to marketing and customer relationships management are completely different from the ones related to production management and coordination. Hence, it is extremely unlikely that a unique entity has the specialized knowledge to effectively perform such different activities at the same time.

A multi-role architecture can be particularly useful to overcome the drawbacks of the broker-centered approach.

In the next Section will be introduced the Enabler-Catalyst-Broker (ECB) configuration, based on the three complementary roles of enabler, catalyst and broker.

\section{THE ECB FRAMEWORK FOR THE VIRTUAL ENTERPRISE}

The ECB is an organizational configuration based on three key roles that the member organizations may play in the virtual enterprise: (a) organization enabler, (b) process catalyst, and (c) broker. In addition to these entities, fundamental roles are played by small and medium enterprises joining the virtual enterprise and by customers.

In the ECB approach, all the members have the same importance. The broker is not most important role. Every phase of the life of a virtual enterprise is leaded by a "primus inter pares" (broker or catalyst). Roles are not fixed: each SME may play different roles in different virtual enterprises. The only "super partes", fixed entity is the enabler, which sets the sub-environment and enables virtual enterprises to be created. The enabler can be seen as an "infrastructure manager".

The dynamic allocation of roles allows a higher potential in identifying business opportunities and ensures that authorities and responsibilities are assigned to the 
subjects more specialized in the particular job to be exploited. In addition, no longer exists any "vital" member, being the network dynamic and members-independent (maybe the only critical entity is the enabler, but it is stable by definition).

Table 1: Main Functions of the Three Key Actors

\begin{tabular}{|c|c|}
\hline Subject & Wunctions \\
\hline Enabler & $\begin{array}{l}\text { - Increases inter-organizational trust } \\
\text { - Develops an on-line environment to let SMEs get in touch } \\
\text { - } \text { Allows on-line negotiation } \\
\text { - Supports Workflow schema design } \\
\text { busports the reconfiguration of virtual enterprise for new }\end{array}$ \\
\hline Catalyst & $\begin{array}{l}\text { - Identifies the business opportunity and envisions the V.E. } \\
\text { - Designs the virtual process at high level } \\
\text { - Manages all aspects related to customers }\end{array}$ \\
\hline Broker & $\begin{array}{l}\text { - Identifies and selects sub-processes to be integrated } \\
\text { - Refines the top-down WF schema and mange exception handling } \\
\text { - Enacts the virtual process } \\
\text { - Coordinates tasks, actors, and applications } \\
\text { - Controls and manages the virtual process enactment }\end{array}$ \\
\hline
\end{tabular}

\subsection{The Enabler}

The enabler is the actor in charge of the design, management and promotion of an on-line environment aimed at supporting the development of cooperation among participating SMEs. The enabler has to create the humus to enable the development of virtual enterprises, an environment similar to a holonic system (Merli and Saccani 1994). In this competitive environment, enterprises (having competitive and complementary competencies) would be able to efficiently and effectively integrate themselves. In addition, they should share a common business culture and should be oriented and motivated towards cooperation.

The enabler is necessary because (unlike participating SMEs that are dynamic and potentially not continuously joining the sub-environment) it is a stable entity, hence able to set the rules and the standards of any cooperation and to provide added value services. It would, in addition to manage the sub-environment, propose customizable methods and tools to efficiently set-up a virtual enterprise (e.g. communication protocols, data sharing methods, workflow design schemas, best practices).

It would also collect, manage and make accessible information about participating SMEs to allow them to easily find partnership opportunities.

Finally, the enabler provides a whole set of added value services to enable enterprises to:

- Meet: to develop a trustworthy climate, enterprises should be able to interact even before and beyond an actual business opportunity.

- Negotiate: once the business opportunity is recognized and the partners selected, it is necessary to support the negotiation phase, to make it lean and rapid. 
- Cooperate: methods and tools to support the design and enactment of the virtual process have to be supplied.

- Disconnect: in a similar environment, it is fundamental that the switching costs are so low as to allow the efficient disconnection and different connection of SMEs. The development of technological and ontological standard is the necessary.

\subsection{The Catalyst}

The enterprise identifying the business opportunity and initiating the virtual enterprise becomes the catalyst. This implies that every enterprise within the competitive sub-environment managed by the broker is potentially able to be a catalyst. It appears to the market as the only supplier of the product/service made by the virtual enterprise: it is similar to the firewall in PC nets, preventing the world from seeing what is happening behind it. Once the process of virtual enterprise creation has been initiated, the catalyst has two possibilities: 1) to become a broker (organizing and managing the virtual enterprise); or 2) to select a broker enterprise within the competitive sub-environment, allowing it to manage the virtual process. The catalyst could be the unique interface between the virtual enterprise and the (external) market, by taking care of developing business plans and all the activities oriented to the market. Post-selling support and warranty services will be managed by the catalyst (eventually, it could initiate a new virtual enterprise to this end). The catalyst designs the virtual process at high level (with a top-down approach starting from the market needs) and generally envisions the configuration of the virtual enterprise.

\subsection{The Broker}

It is the entity in charge of the coordination, management, and control of the virtual process, at the same time, it has to allow virtual enterprise members to access the knowledge base supplied by each of them (preventing unauthorized access to critical, not shared resources). The broker translates the customer requirements and the top-down workflow schema developed by the catalyst into operative specifications. It selects, organizes, and synchronizes the (sub)processes; its interactions with the catalyst are continuous and very close (in particular during the start-up phase).

The broker has a fundamental role in supporting the integration of the members of the virtual enterprise. To this end, it has three key functions:

1) Process selection: it identifies and selects the sub-processes to be integrated into one virtual process (on the bases of the cooperation schema designed by the catalyst).

2) Workflow schema refinement: it develops a bottom-up workflow schema (starting from selected elementary processes) in accordance with the topdown schema designed by the catalyst. The conceptual schema of the virtual schema will contain also alternative flows of tasks to cope with likely exceptions. As for not very likely ones, the broker will define and assign the recovery actions for any class of exceptions. 
3) Virtual process enactment and management: it manages and controls the enactment of the virtual process, monitoring the performances and coordinating the exception handling tasks.

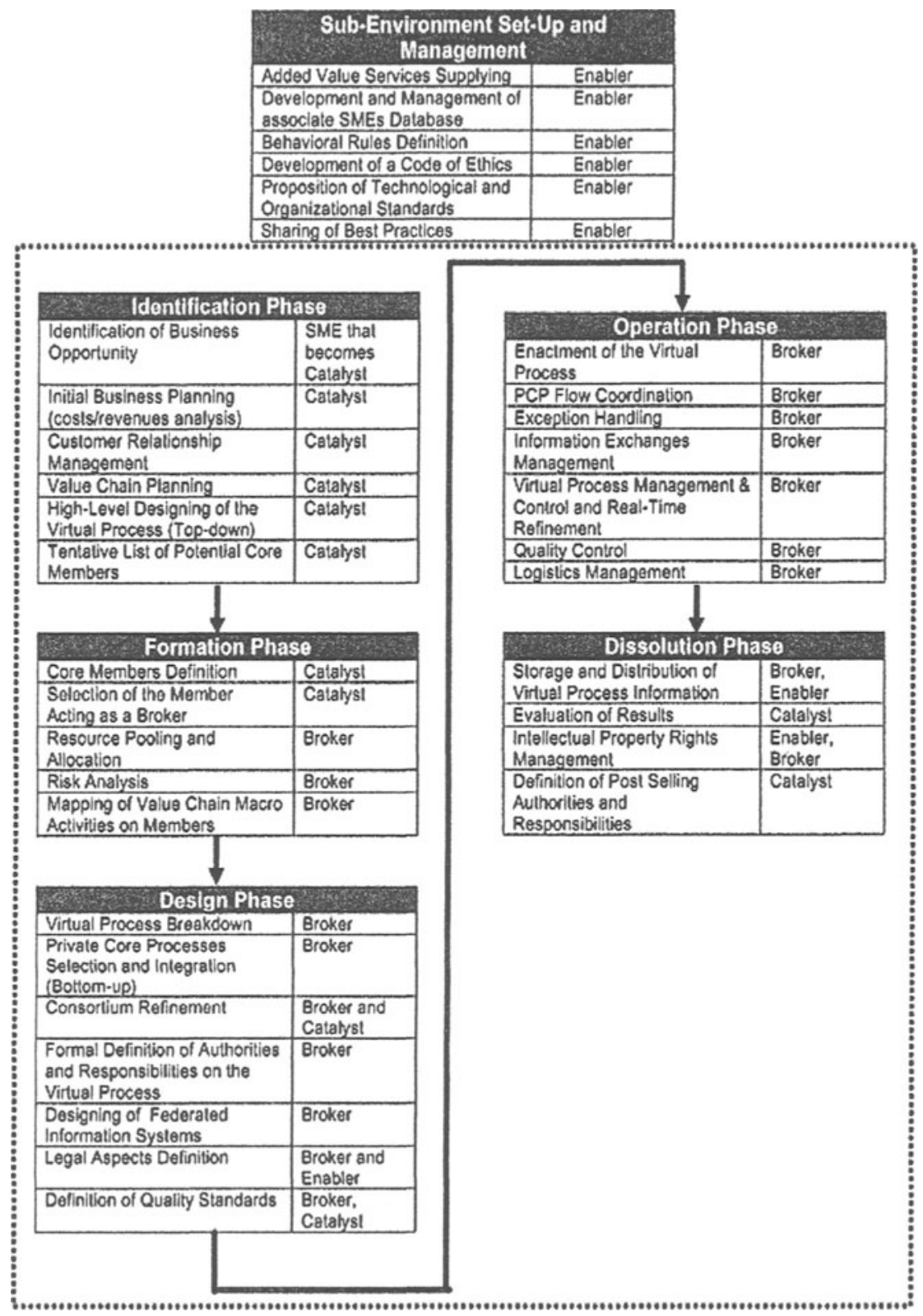

Figure 1: Life Cycle of a Virtual Enterprise 


\section{CONCLUSION AND FURTHER RESEARCH}

In this paper, we presented the preliminary results of an ongoing research aimed at developing an organizational framework for virtual enterprises. The ECB Framework could be useful in managing temporary networks of enterprises. The definition of dynamic roles allows a continuous reorganization of the virtual enterprise. The proposed framework, that is here provided with respect to methodological aspects, needs to be validated in real world applications. Our research activity is now being headed towards two main themes related to this framework:

- Organizational Framework Testing: the architecture based on enabler, catalyst and broker is now being applied in the field of virtual trade fairs.

- Implementation of and inter-organizational workflow schema.

\section{ACKNOWLEDGEMENTS}

This work has been partially supported by the Italian Ministry of University and Research, under the Inter-University Research Project "Organization \& Management of Information Systems in Virtual Enterprises within Digital Marketplaces".

\section{REFERENCES}

BIDSAVER, IST Project 1999-10768. http://www.ceconsulting.it/ve/bidsaver.html

BREMER et al. (1999). Global Virtual Business - A Systematic Approach for Exploiting Business Opportunities in Dynamic Markets. International Journal of Agile Manufacturing, vol.2, issue 1.

CAMARINHA-MATOS, L.M. et al. (eds.) (2000). E-Business and Virtual Enterprises - Managing Business-to-Business Cooperation. Kluwer Academic Publishers, IFIP Vol. 184.

COSME Project. http://veforum.Irt.unibw-muenchen.de/forum/ThirdLevel/gve.html

COYNE, K.P. and R. DYE (1998). The Competitive Dynamics of Network-Based Businesses. Harvard Business Review, January-February, 99-109.

DAVENPORT, T.H. (1993). Process Innovation. Reengineering Work through IT. Harvard Business School Press, Boston.

D'ATRI, A. et al. (eds.) (2001). Open Enterprise Solutions: Systems, Experiences, and Organizations. Luiss Edizioni, Rome.

DESANCTIS, G. and P. MONGE (1999). Introduction to the Special Issue: Communication Processes for Virtual Organizations. Organization Science, vol.10, no. 6, November-December, 693-703.

FRANKE, U.J. (2001). The Concept of Virtual Web Organisations and its Implications on Changing Market Conditions. Electronic Journal of Organizational Virtualness, vol.3, n.4, 43-64.

MALONE, T.W. and K. CROWSTON (1994). Toward an Interdisciplinary Theory of Coordination. Computing Surveys, vol.26, n.1.

MALONE , T.W. et al. (1999). Tools for Inventing Organizations: Toward a Handbook for Organizational Processes. Management Sciences, vol.45, no. 3, 425-443.

MERLI, G. and C. SACCANI (1994). L'Azienda Olonico-Virtuale. Il Sole 24 Ore Libri, Milan.

MOWSHOVITZ, A. (1997). Virtual Organization. Communication of the ACM, Special issue on Virtual Organizations, vol.40, no. 9, September.

PRODNET ESPRIT Project 22.647. http://www.uninova.pt/ prodnet/

ROCKART, J.F. and J.E. SHORT (1991). The Networked Organization. In SCOTT MORTON, S.M. (ed.) (1991). The Corporation of the 1990s. Oxford University Press, New York, 189-219.

VENKATRAMAN, N. (1994). IT-Enabled Business Transformation: from Automation to Business Scope Redefinition. Sloan Management Review, Winter, 73-78.

VIVE Initiative. http://www.vive-ig.net/ve_default.htm

WIGAND, R. et al. (1999). Information Organization and Management. John Wiley and Sons, New York. 